\title{
Emotion Mining in Social Media Data
}

\author{
Jaishree Ranganathan and Angelina Tzacheva \\ UNC Charlotte, Charlotte, North Carolina, 28223, USA, \\ \{jrangan1, aatzache\}@uncc.edu,
}

\begin{abstract}
Emotions are known to influence the perception of human beings along with their memory, thinking and imagination. Human perception is important in today's world in a wide range of factors including but not limited to business, education, art, and music. Microblogging and Social networking sites like Twitter, Facebook are challenging sources of information that allow people to share their feelings and thoughts on a daily basis. In this paper we propose an approach to automatically detect emotions on Twitter messages that explores characteristics of the tweets and the writer's emotion using Support Vector Machine LibLinear model and achieve 98\% accuracy. Emotion mining gained attraction in the field of computer science due to the vast variety of systems that can be developed and promising applications like remote health care system, customer care services, smart phones that react based on users's emotion, vehicles that sense emotion of the driver. These emotions help understand the current state of user. In order to perform suitable actions or provide suggestions on how user's can enhance their feeling for a better healthy life-style we use actionable recommendations. In this work we extract action rules with respect to the user emotions that help provide suggestions for user's.
\end{abstract}

Keywords: Data Mining, Emotion, Microblog, Sentiment Analysis, Twitter.

\section{Introduction}

Emotion is a combinatorial result of a persons evaluation of a situation along with the physiological arousal [1]. Classification of human emotions has been long under research since the early 60 's. One of the earliest studies [2] suggests 'anger', 'fear', 'enjoyment', 'sadness', 'disgust', 'surprise' as the basic emotions Fig. 1 universal for all human beings. Any kind of information available holds a 'meaning' to it. Meaning has associated Connotation and Denotation. Denotation is the dictionary meaning, while Connotation is the emotional association to the information. For instance the words 'childish' and 'childlike' both have identical denotations, whereas the former has a more insulting connotation when refered to a person and the latter has more positive meaning. According to researchers Connotation has gained more attention with psychology. Thus emotion plays an important role in our understanding of the information entities in day to day life. Internet naturally poses a wider platform for expressing this kind

Dhinaharan Nagamalai et al. (Eds) : CCSEIT, ICBB, DMDB, AIAP, CNSA - 2019

pp. 99-111, 2019. (C) CS \& IT-CSCP 2019

DOI: $10.5121 /$ csit.2019.90609 
of emotional information. One such prominent platform is 'Social Media' which includes online social networks like 'Facebook' and microblogs like 'Twitter' [3].

We use the dataset formed of messages collected from the popular microblogging platform Twitter. Users of this platform tend to share their opinion or feelings about political events, natural disaster, products and companies, environment, community and much more on a day-to-day basis. This kind of user information is of interest to different communities. For instance, tweets about environment, community from a particular county and state will help the government officials understand the standard of living of people. This helps them make better amendments to the policies for the well being of the people. In this paper we utilize Weka Affective Tweets package [4] to explore the tweet text characteristics such as the lexicon features, semantic features and word embeddings which are considered to improve the classification model performance [5], [6], [7].

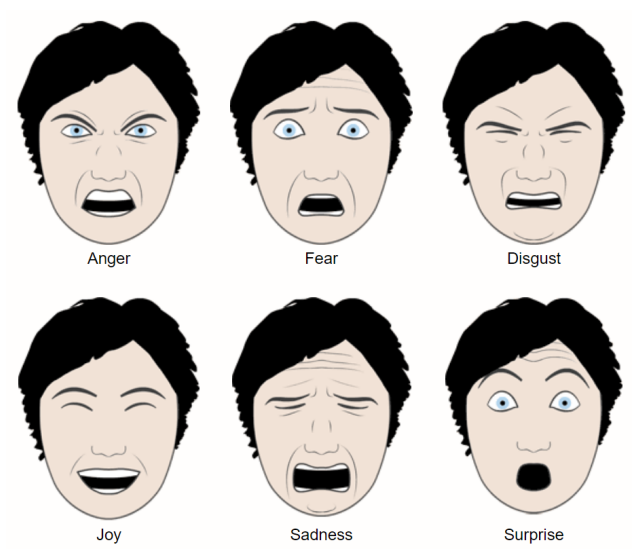

Fig. 1. Basic Emotions - Facial Expression ${ }^{[8]}$

Knowledge Discovery is the process of extracting interesting patterns and applying such patterns to specific areas of interest. To find such interesting patterns from data there are wide range of techniques likes classification, rule mining, clustering etc. Source of data in the era of internet has become huge with five V's (Volume, Variety and Velocity, Veracity and Value)of defining big data. With such huge amount of data being generated from variety of sources it is obvious that the discovered knowledge might not be of much use to applications which are in need of specific suggestions including smart phones, remote health care systems, customer care services, pedagogical approaches for teaching (student teacher evaluation), e-learning and other such applications. One of the major problem in the knowledge discovery process is reducing the volume of discovered patterns and selecting the appropriate interestingness measure [9]. One of the techniques to extract appropriate interseting patterns is action rules proposed 
by authors [10]. Action rules are special types of rules which forms a hint to the users, show a way to reclassify objects with respect to some distinguished attribute called the decision attribute. Emotion mining gained attraction in the field of computer science due to the vast variety of systems that can be developed and promising applications like remote health care system, customer care services, smart phones that react based on users's emotion, vehicles that sense emotion of the driver. Emotions mined from text data is vast, in order to make sensible suggestions we use action rules that help suggest ways to alter the user's emotion to a better or more positive state.

In this work we automatically detect user emotion from tweet data using the NRC Emotion Lexicon [11], [12] to label the Emotion class for our data. We use Support Vector Machine LibLinear implementation and features including word n-grams, character n-grams, brown word clusters, and part-of-speech tags. Also we extract action rules to identify what factors can be improved in order for a user to attain more desirable positive emotion or feeling.

\section{Related Work}

Sentiment Analysis involves use of natural language processing to identify and extract sentiment or opinion. There is a wide range of literature covering the areas of text sentiment analysis which aims to classify the data with polarity and also into further fine grained emotion. In this section we include related works in the area of sentiment mining from text.

\subsection{Sentiment and Emotion Mining}

Authors Bruyne et al. [6] use ensemble of classifiers for the multi-class multi-label problem. They utilize different aspects of the tweet by extracting tweet features including lexicon features, n-gram features, syntactic, semantic features, and word embeddings. They suggest that classifier chain performs better than the individual binary classifiers.

Authors Himeno et al. [7] use convolutional neural networks to classify the tweet emotion intensity. Their idea is based on the observation that n-grams have vital effects to represent the tweet emotion. They achieve an average correlation co-efficient of 0.620 .

Authors Turcu et al. [13] use supervised machine learning models like Naive Bayes, K-Nearest Neighbor and Support Vector Macines, and deep learning neural net tensor flow model, decision tree for affective tweets task and achieve best accuracy of approximately 0.73 for emotion Joy.

Authors Mishne et al. [14] use blog text data to classify the writer's emotion or mood. They train the SVMlight model of Support Vector Mahine using significant features extracted from the blog data. The features include frequency counts (words, Part-Of-Speech), and length of blog post; subjective nature of blogs like semantic orientation, Point-wise Mutual Information (PMI) which is a measure of the degree of association between two terms; features unique to 
online text like emphasized words, special symbols including punctuation's, and emoticons.

Authors Yassine and Hajj [15] use undupervised techniques on facebook data to study the friendship relations and emotions expression in online social networks. Similarly authors Sun et al. [16] use sentiment feedback on social media data to improve item recommendations. They validate different approaches including Support Vector Machines (SVM), SVM-Boosting, Naive Bayes and others along with the proposed ensemble learning-based sentiment classification method. It is observed that SVM method achieves best performance among all the machine learning classifiers for sentiment detection, but the proposed ensemble method further helps to overcome the challenges associated with special features of affective text and achieves an accuracy of $86.7 \%$.

\subsection{Actionable Pattern Mining and Applications}

Actionability is a property of the discovered knowledge. If user's can act upon a pattern and benefit from the action, these patterns are considered as actionable patterns. This section reviews literature for actionable pattern mining.

Action Rules are used to discover patterns in the form of rules called decision rules 'if ..., then...' [17]. These patterns provide details about events in the past and suggestions for making prospective decisions. In medical field, for disease diagnosis, decision rules help identify the correlation between symptoms and sickness with the past data and help diagnose new patients. Another prospective usefulness of decision rules is getting the desired effect on dependent variables by building strategy of intervention on the independent variables. In the medical example, this can be explained as modifying symptoms or treating symptoms to cure sickness.

Authors Tzacheva et al. [18], discover low cost actionable patterns and recommendations in distributed environment. They use the algorithm described by [19] and develop the system using Apache Spark framework and Hadoop Distributed File System (HDFS) for scalability and efficiency. They evaluate the approach using car evaluation dataset, mammographic dataset and achieve best results.

In relation to Emotion mining, Actionable patterns may suggest a way to alter the user's emotion from a negative, or neutral to a more positive Emotion, or a desirable state / attitude. For example, for customer care services, recommendation systems for online shopping, or smart phones that are able to recognize human emotions, Emotion altering Actionable Patterns include: suggesting calming music, playing mood enhancing movie, changing the background colors to suiting ones, or calling caring friends (for smart phones). In [20] the primary intent of the Action Rules generated is to provide viable suggestions on how to make a twitter user feel more positive. For Twitter social network data, Actionable Recommendations may include - how to increase user's friends count, how to increase the user's follower's count, and how to change the overall sentiment from negative to positive, or from neutral to positive. 


\section{Methodology}

\subsection{Data Collection}

Twitter streaming API provides a push of data as tweets happen in near realtime. In this study we use the Twitter API to collect around 520,000 tweets as raw data. The data includes the following attributes TweetID, ReTweetCount, TweetFavouriteCount, TweetText, TweetLanguage, TweetSource, UserID, UserFollowersCount, UserFavouritesCount, UserFriendsCount, UserLocation, MediaEntities. The overall methodology is shown in Fig .2.

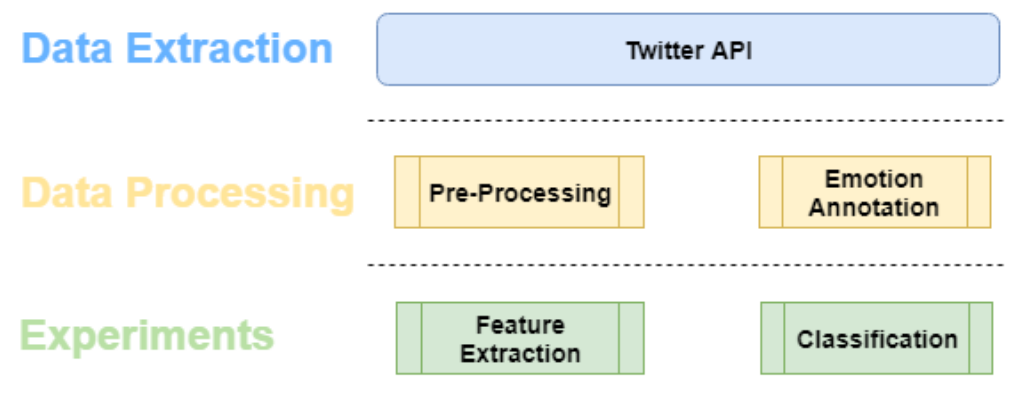

Fig. 2. Methodology.

\subsection{Pre-Processing and Emotion Annotation}

Tweets are generally informal and not syntactically well formed which can be attributed to limitation of 140 characters. So the raw tweets need considerable pre-processing before further steps of emotion labeling, feature extraction and classification. We lowercase all the letters in the tweet, remove stop words, replace slang words with formal text, example b4 $\rightarrow$ before, chk $\rightarrow$ check etc. After pre-processing we have around 200,000 tweets.

As part of emotion labeling we use the National Research Council - NRC Emotion lexicon [11], [12], [21], [22] . The Annotations in the lexicon are at WORD-SENSE level. Each line has the format: $<$ Term $><$ AffectCategory $>$ $<$ AssociationFlag $>$ as shown in Fig. 3. Each tweet in the dataset is annotated with the corresponding emotion based on the weightage computed using features such as word-sense, hashtags and emoticons. Thus we obtain annotated dataset where each record is labeled with coressponding emotions such as 'anger', 'fear', 'disgust', 'sadness', 'anticipation', 'joy', and 'trust'. In the process of annotation additional score features are added to the dataset which helps in the classification process. 


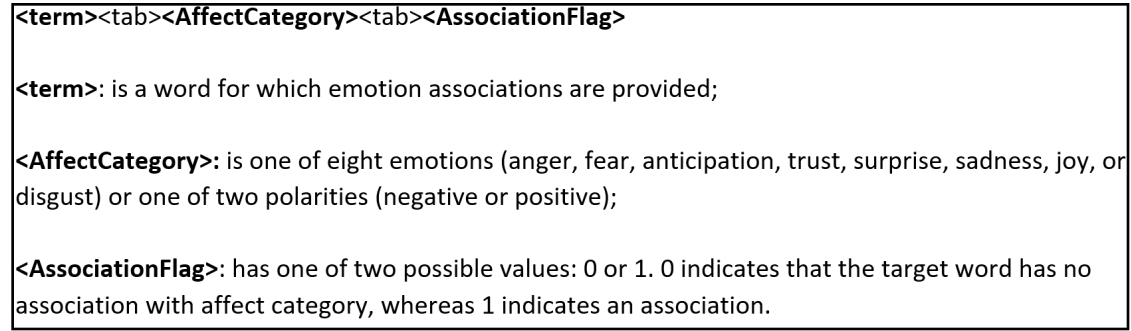

Fig. 3. NRC Lexicon Word Level Annotation.

\subsection{Tweet Feature extraction}

In the literature wide range of features have been explored in the task of tweet sentiment analysis including unigrams, bigrams, n-grams, part-of-speech (POS) tags, word embedding, word clusters [23], [24], [25], [26], [7], [27]. In this work we use TweetToSparseFeatureVector filter in Weka Affective tweets [4] package to extract word n-grams, character n-grams, brown word clusters and part-ofspeech tags. The dataset with the following attributes is passed to the Weka data mining software AngerScore, TrustScore, FearScore, SadnessScore, AnticipationScore, DisgustScore, SurpriseScore, JoyScore, PositiveScore, NegativeScore, TweetLanguage, TweetSource, UserFollowersCount, UserFavoritesCount, UserFriendsCount, UserLanguage, MediaEntities, FinalEmotion, TweetTokens.

\subsection{Classification}

Classification is a supervised machine learning model that learns the data using labeled train set and predicts the test set for which the model does not know the actual class labels. This model is further evaluated with the help of validation measures like precision, recall, f1-measure, and accuracy. There are a wide range of classification models available including Decision Trees, Naive Bayes, Support Vector Machines, K Nearest Neighbor, Rule based classifier and many more. Support Vector Machine (SVM) is considered to be one of the powerful learning method for unstructured data like text because of its generalization ability [28]. In this paper we have used Support Vector Machine LibLinear [29] as a classification model for automatically classifying Twitter dataset with fine grained emotion. LibLinear is a open source package that is considered to be efficient for training large-scale problems. We use the data mining software WEKA (Waikato Environment for Knowledge Analysis) [30] for emotion classification.

\subsection{Actionable Pattern Discovery}

Actionability is a property of the discovered knowledge. Patterns are considered Actionable if the user can act upon them, and if this action can benefit the user, or help them to accomplish their goals. Action Rules mining is a method to 
extract Actionable patterns from the data. Action Rules are rules that describe a possible transition of data from one state to another more desirable state. The formal definition of action rules as described in [9] is given in definition 1.

Definition 1. By an Action Rule we mean an expression $r=\left[t_{1} \rightarrow t_{2}\right]$, where $t_{1}$ is an action term, and $t_{2}$ is an atomic action term.

Additionally, we assume, that $\operatorname{Dom}\left(t_{2}\right)=\{d\}$ and $\operatorname{Dom}\left(t_{1}\right) \subseteq A$, where $A$ is a set of attributes.

The domain Dom(r) of Action Rule $r$ is defined as Dom $\left(t_{1}\right) \cup \operatorname{Dom}\left(t_{2}\right)$.

Table 1. Information System S.

\begin{tabular}{rrrrr}
\hline $\mathrm{X}$ & Attribute a Attribute $\mathrm{b}$ Attribute c Attribute d \\
\hline $\mathrm{x}_{1}$ & $\mathrm{a}_{1}$ & $\mathrm{~b}_{1}$ & $\mathrm{c}_{1}$ & $\mathrm{H}$ \\
$\mathrm{x}_{2}$ & $\mathrm{a}_{2}$ & $\mathrm{~b}_{2}$ & $\mathrm{c}_{1}$ & $\mathrm{H}$ \\
$\mathrm{x}_{3}$ & $\mathrm{a}_{2}$ & $\mathrm{~b}_{1}$ & $\mathrm{c}_{1}$ & $\mathrm{~A}$ \\
$\mathrm{x}_{4}$ & $\mathrm{a}_{1}$ & $\mathrm{~b}_{1}$ & $\mathrm{c}_{2}$ & $\mathrm{~A}$ \\
$\mathrm{x}_{5}$ & $\mathrm{a}_{2}$ & $\mathrm{~b}_{1}$ & $\mathrm{c}_{2}$ & $\mathrm{~A}$ \\
$\mathrm{x}_{6}$ & $\mathrm{a}_{2}$ & $\mathrm{~b}_{2}$ & $\mathrm{c}_{2}$ & $\mathrm{H}$ \\
$\mathrm{x}_{7}$ & $\mathrm{a}_{1}$ & $\mathrm{~b}_{1}$ & $\mathrm{c}_{2}$ & $\mathrm{~A}$ \\
$\mathrm{x}_{8}$ & $\mathrm{a}_{1}$ & $\mathrm{~b}_{2}$ & $\mathrm{c}_{1}$ & $\mathrm{~A}$ \\
$\mathrm{x}_{9}$ & $\mathrm{a}_{1}$ & $\mathrm{~b}_{1}$ & $\mathrm{c}_{1}$ & $\mathrm{H}$ \\
$\mathrm{x}_{10}$ & $\mathrm{a}_{2}$ & $\mathrm{~b}_{2}$ & $\mathrm{c}_{1}$ & $\mathrm{H}$ \\
\hline
\end{tabular}

Consider the information system $\mathrm{S}$ in Table 1. Equation (1) and (2) are example Action Rules. According to equation (1) $r_{1}$ says that if the value $a_{2}$ remains unchanged and value $\mathrm{b}$ will change from $b_{2}$ to $b_{1}$ for a given object $x$, then it is expected that the value $d$ will change from $H$ to $A$ for object $x$. Clearly, $\operatorname{Dom}\left(r_{1}\right)=\{a, b, d\}$. In a similar way, the rule $r_{2}$ in equation (2) says that if the value $c_{2}$ remains unchanged and value $b$ will change from $b_{2}$ to $b_{1}$, then it is expected that the value $d$ will change from $H$ to $A$, and $\operatorname{Dom}\left(r_{2}\right)=\{b, c, d\}$.

$$
\begin{aligned}
& r_{1}=\left[\left(\left(a, a_{2} *\left(b, b_{2} \rightarrow b_{1}\right)\right) \rightarrow(d, H \rightarrow A)\right]\right. \\
& r_{2}=\left[\left(\left(c, c_{2} *\left(b, b_{2} \rightarrow b_{1}\right)\right) \rightarrow(d, H \rightarrow A)\right]\right.
\end{aligned}
$$

Definition 2. Standard interpretation $N_{s}$ of action terms in $S=(X, A, V)$ is defined as follows:

1. If $\left(a, a_{1} \rightarrow a_{2}\right)$ is an atomic term, then

$N_{s}\left(\left(a, a_{1} \rightarrow a_{2}\right)\right)=\left[\left\{x \in X: a(x)=a_{1}\right\},\left\{x \in X: a(x)=a_{2}\right\}\right]$

2. If $t_{1}=\left(a, a_{1} \rightarrow a_{2}\right) * t$ and $N_{s}(t)=\left[Y_{1}, Y_{2}\right]$, then

$N_{s}\left(t_{1}\right)=\left[Y_{1} \cap\left\{x \in X: a(x)=a_{1}\right\}, Y_{2} \cap\left\{x \in X: a(x)=a_{2}\right\}\right]$. 
Now we define $\left[Y_{1}, Y_{2}\right] \cap\left[Z_{1}, Z_{2}\right]$ as $\left[Y_{1} \cap Z_{1}, Y_{2} \cap Z_{2}\right]$.

Assume that $N_{s}\left(t_{1}\right)=\left[Y_{1}, Y_{2}\right]$ and $N_{s}\left(t_{2}\right)=\left[Z_{1}, Z_{2}\right]$.

Then $N_{s}\left(t_{1} * t_{2}\right)=N_{s}\left(t_{1}\right) \cap N_{s}\left(t_{2}\right)$. Let $r=\left[t_{1} \rightarrow t_{2}\right]$ be an Action Rule, where $N_{s}\left(t_{1}\right)=\left[Y_{1}, Y_{2}\right], N_{s}\left(t_{2}\right)=\left[Z_{1}, Z_{2}\right]$.

Definition 3. By support and confidence of rule $r$ we mean:

1. $\sup (r)=\min \left\{\operatorname{card}\left(Y_{1} \cap Z_{1}\right), \operatorname{card}\left(Y_{2} \cap Z_{2}\right)\right\}$

2. $\operatorname{conf}(r)=\frac{\operatorname{card}\left(Y_{1} \cap Z_{1}\right)}{\operatorname{card}\left(Y_{1}\right)}$. $\frac{\operatorname{card}\left(Y_{2} \cap Z_{2}\right)}{\operatorname{card}\left(Y_{2}\right)}$

if $\operatorname{card}\left(Y_{1}\right) \neq 0, \operatorname{card}\left(Y_{2}\right) \neq 0, \operatorname{card}\left(Y_{1} \cap Z_{1}\right) \neq 0, \operatorname{card}\left(Y_{2} \cap Z_{2}\right) \neq 0$

3. $\operatorname{conf}(r)=0$ otherwise

Now, let us consider the equation (1) for support and confidence with example. For the rule $\mathrm{r}_{1}$ we have:

$-N_{s}\left(a, a_{2} \rightarrow a_{2}\right)=\left[\left\{x_{2}, x_{3}, x_{5}, x_{6}, x_{10}\right\},\left\{x_{2}, x_{3}, x_{5}, x_{6}, x_{10}\right\}\right]$

$-N_{s}\left(b, b_{2} \rightarrow b_{1}\right)=\left[\left\{x_{2}, x_{6}, x_{8}, x_{10}\right\},\left\{x_{1}, x_{3}, x_{4}, x_{5}, x_{7}, x_{9}\right\}\right]$

$-N_{s}\left(a, a_{2} \rightarrow a_{2}\right) *\left(b, b_{2} \rightarrow b_{1}\right)=\left[\left\{x_{2}, x_{6}, x_{10}\right\},\left\{x_{3}, x_{5}\right\}\right]$

$-N_{s}(d, H \rightarrow A)=\left[\left\{x_{1}, x_{2}, x_{6}, x_{9}, x_{10}\right\},\left\{x_{3}, x_{4}, x_{5}, x_{7}, x_{8}\right\}\right]$

Therefore, for rule $r_{1}$, support $\sup \left(r_{1}\right)=2$, confidence $\operatorname{conf}\left(r_{1}\right)=\frac{3}{3} \cdot \frac{2}{2}=1$.

In this work we use the method proposed by authors bagavathi et al. [31] to extract action rules for the emotion labeled Twitter dataset. The method 2 in [31] is to extract action rules by vertical split of the data. This method utilizes association action rules [32] which follows iterative method to extract all possible action rules. In order to overcome the computational complexity and expense, authors in [31] propose vertical data split method for faster computation and parallel processing.

\section{Experiments and Results}

\subsection{Classification - Support Vector Machine}

For experiments we use the processed dataset and classify using SVM LibLinear [29] classification model. The classifier excludes the string type attribute, in this case the TweetTokens which is the text of the tweet. This is because the text is processed in the feature extraction step to get additional attributes like word n-grams, brown word clusters, and part-of-speech tags. Thus we achieve an accuracy of $98 \%$. The confusion matrix and precision, recall, f-measure is shown in Table. 2., and Table. 3 respectively.

\subsection{Action Rules}

Inorder to identify what changes in attributes lead to change in emotion to a better positive state we extract action rules from the emotion labeled dataset. The continous data is discretized to intervals with the help of WEKA data mining software using unsupervised attribute discretization. Attributes AngerScore, TrustScore, FearScore, SadnessScore, AnticipationScore, DisgustScore, 
Table 2. Confusion Matrix.

\begin{tabular}{rrrrrrrrr}
\hline $\mathrm{a}$ & $\mathrm{b}$ & $\mathrm{c}$ & $\mathrm{d}$ & $\mathrm{e}$ & $\mathrm{f}$ & $\mathrm{g}$ & $\mathrm{h}$ & Classified As \\
\hline 15449 & 16 & 0 & 0 & 0 & 1 & 7 & 0 & $\mathbf{a}$ - sadness \\
2 & 10259 & 1 & 127 & 7 & 4 & 1 & 7 & $\mathbf{b}-$ joy \\
44 & 4 & 3057 & 3 & 6 & 8 & 14 & 11 & c - fear \\
9 & 12 & 0 & 20411 & 4 & 0 & 12 & 1 d - anticipation \\
77 & 43 & 2 & 49 & 8990 & 11 & 10 & 5 & e - trust \\
57 & 16 & 9 & 6 & 12 & 2155 & 1 & 7 & $\mathbf{f}$ - surprise \\
21 & 5 & 0 & 0 & 1 & 0 & 4100 & 26 & g - anger \\
16 & 22 & 4 & 1 & 1 & 2 & 8 & 4741 & $\mathbf{h}$ - disgust \\
\hline
\end{tabular}

Table 3. Precision, Recall, F-Measure.

\begin{tabular}{rrrr}
\hline Precision & \multicolumn{2}{r}{ Recall F-Measure } & Class \\
\hline 0.986 & 0.998 & 0.992 & sadness \\
0.989 & 0.986 & 0.987 & joy \\
0.995 & 0.971 & 0.983 & fear \\
0.991 & 0.998 & 0.995 & anticipation \\
0.997 & 0.979 & 0.987 & trust \\
0.988 & 0.952 & 0.970 & surprise \\
0.987 & 0.987 & 0.987 & anger \\
0.988 & 0.989 & 0.988 & disgust \\
\hline
\end{tabular}


SurpriseScore, JoyScore, PositiveScore, NegativeScore, LoveScore, PeopleScore, MessageScore, UserFollower- sCount, UserFavoritesCount, UserFriendsCount, Tweet-Source, FinalEmotion from the original dataset are used experimental

purpose. The dataset with 174688 instances is divided into 100 parts based on the target attribute 'FinalEmotion'. Action rules are generated for one part of teh dataset. The sample action rules are shown in Fig. 4.

\begin{tabular}{|l|l|}
\hline AR1 & $\begin{array}{l}(\text { AnticipationScore, } 0->0)^{\wedge}(\text { DisgustScore, } 0->0)^{\wedge}(\text { LOVESCORE, } 0->1)^{\wedge}(\text { SadnessScore, } 2->0)^{\wedge} \\
(\text { GOINGSCORE, } 0->0)=>(\text { FinalEmotion, sadness }->\text { joy) }[\text { Support:-53.0, Confidence:-93.23308270676691\%] }\end{array}$ \\
\hline AR2 & $\begin{array}{l}\left.\text { (AnticipationScore, } 0->0)^{\wedge}(\text { NegativeScore, } 1->0)^{\wedge} \text { (SadnessScore, } 2->0\right)^{\wedge}(\text { TrustScore, } 0->0)^{\wedge} \\
(\text { INSTANTSCORE, } 0->0)^{\wedge}(\text { KNOWSCORE, } 0->0)^{\wedge}(\text { TweetSource, } 1->1)=>(\text { FinalEmotion, sadness }->\text { joy) } \\
{[\text { Support:-61.0, Confidence:-79.41828254847645\%] }}\end{array}$ \\
\end{tabular}

Fig. 4. Sample Action rules.

Let us consider the action rule AR1, this rule suggest possible chnges to achiece a desirable emotional state of 'joy'. If user tends to use more positive words as denoted by (LOVESCORE, $0 \rightarrow 1)$ and reduce the negative words denoted by (SadnessScore, $2 \rightarrow 0$ ) then it is possible to change the emotion from 'sadness' to 'joy'. In that case, the emotion associated with this user tweet can be classified as 'joy'. This example is more intutive in case of applications where it is required to monitor people emotions in a particular city or county in order to understand the life satisfaction and help in public policy making and societal well-being measures.

\section{Conclusions}

In this work we automatically detect user emotion from tweet data using the NRC Emotion Lexicon [11], [12] to label the Emotion class for our data. We use Support Vector Machine LibLinear implementation and features including word n-grams, character n-grams, brown word clusters, and part-of-speech tags and achieve a $10 \%$ improved accuracy compared to the previous method [33]. Also we extract action rules to identify what factors can be improved in order for a user to attain more desirable positive emotion or feeling. Identifying emotions from text is an emerging area of research and has its roots in many disciplines including Psychology, Neuro Science, Social Science, Computer Science and many more. Systems that identify emotions and suggest actionable recommendations has wode variety of applications like: improving the teaching models and learning outcomes from student evaluations, understanding customer satification surveys and how it can help improve business. 


\section{Bibliography}

[1] C. E. Izard, Human emotions. Springer Science \& Business Media, 2013.

[2] P. Ekman, "An argument for basic emotions," Cognition $\&$ emotion, vol. 6, no. 3-4, pp. 169-200, 1992.

[3] E. Huffman and S. Prentice, "Social media's new role in emergency management," tech. rep., Idaho National Laboratory (INL), 2008.

[4] S. Mohammad and F. Bravo-Marquez, "Emotion intensities in tweets," in Proceedings of the 6th Joint Conference on Lexical and Computational Semantics, *SEM@ACM 2017, Vancouver, Canada, August 3-4,2017, pp.65$77,2017$.

[5] Z. Zhang, Q. Zhou, and H. Wu, "Nlpzzx at semeval-2018 task 1: Using ensemble method for emotion and sentiment intensity determination," in Proceedings of The 12th International Workshop on Semantic Evaluation, pp. 116-122, 2018.

[6] L. De Bruyne, O. De Clercq, and V. Hoste, "Lt3 at semeval-2018 task 1: A classifier chain to detect emotions in tweets," in SemEval-2018, pp. 123-127, Association for Computational Linguistics, 2018.

[7] M. Aono and S. Himeno, "Kde-affect at semeval-2018 task 1: Estimation of affects in tweet by using convolutional neural network for n-gram," in Proceedings of The 12th International Workshop on Semantic Evaluation, pp. 156-161, 2018.

[8] O. Spindler and T. Fadrus, "Grimace project documentation," Vienna University of Technology, 2009.

[9] A. Dardzinska, Action rules mining, vol. 468. Springer, 2012.

[10] Z. W. Ras and A. Wieczorkowska, "Action-rules: How to increase profit of a company," in European Conference on Principles of Data Mining and Knowledge Discovery, pp. 587-592, Springer, 2000.

[11] S. M. Mohammad and P. D. Turney, "Crowdsourcing a word-emotion association lexicon," Computational Intelligence, vol. 29, no. 3, pp. 436-465, 2013.

[12] S. M. Mohammad and P. D. Turney, "Emotions evoked by common words and phrases: Using mechanical turk to create an emotion lexicon," in Proceedings of the NAACL HLT 2010 workshop on computational approaches to analysis and generation of emotion in text, pp. 26-34, Association for Computational Linguistics, 2010.

[13] R.-A. Turcu, S. M. Amarandei, I.-A. Flescan-Lovin-Arseni, D. Gifu, and D. Trandabat, "Emointens tracker at semeval-2018 task 1: Emotional intensity levels in\# tweets," in Proceedings of The 12th International Workshop on Semantic Evaluation, pp. 177-180, 2018.

[14] G. Mishne et al., "Experiments with mood classification in blog posts," in Proceedings of ACM SIGIR 2005 workshop on stylistic analysis of text for information access, vol. 19, pp. 321-327, 2005. 
[15] M. Yassine and H. Hajj, "A framework for emotion mining from text in online social networks," in 2010 IEEE International Conference on Data Mining Workshops, pp. 1136-1142, IEEE, 2010.

[16] J. Sun, G. Wang, X. Cheng, and Y. Fu, "Mining affective text to improve social media item recommendation," Information Processing $\& 3$ Management, vol. 51, no. 4, pp. 444-457, 2015.

[17] S. Greco, B. Matarazzo, N. Pappalardo, and R. Slowinski*, "Measuring expected effects of interventions based on decision rules," Journal of Experimental \& Theoretical Artificial Intelligence, vol. 17, no. 1-2, pp. 103-118, 2005.

[18] A. A. Tzacheva, A. Bagavathi, and L. Ayila, "Discovery of action rules at lowest cost in spark," in Data Mining Workshops (ICDMW), 2017 IEEE International Conference on, pp. 87-94, IEEE, 2017.

[19] A. Bagavathi and A. A. Tzacheva, "Rule based systems in distributed environment: Survey," in Proceedings of International Conference on Cloud Computing and Applications (CCA17), 3rd World Congress on Electrical Engineering and Computer Systems and Science (EECSS'17), pp. 1-17, 2017.

[20] J. Ranganathan, A. S. Irudayaraj, and A. A. Tzacheva, "Action rules for sentiment analysis on twitter data using spark," in Data Mining Workshops (ICDMW), 2017 IEEE International Conference on, pp. 51-60, IEEE, 2017.

[21] S. M. Mohammad and S. Kiritchenko, "Using hashtags to capture fine emotion categories from tweets," Computational Intelligence, vol. 31, no. 2, pp. 301-326, 2015.

[22] S. M. Mohammad, "\# emotional tweets," in Proceedings of the First Joint Conference on Lexical and Computational Semantics-Volume 1: Proceedings of the main conference and the shared task, and Volume 2: Proceedings of the Sixth International Workshop on Semantic Evaluation, pp. 246-255, Association for Computational Linguistics, 2012.

[23] B. Pang and L. Lee, "A sentimental education: Sentiment analysis using subjectivity summarization based on minimum cuts," in Proceedings of the 42nd annual meeting on Association for Computational Linguistics, p. 271, Association for Computational Linguistics, 2004.

[24] B. Pang, L. Lee, and S. Vaithyanathan, "Thumbs up?: sentiment classification using machine learning techniques," in Proceedings of the ACL-02 conference on Empirical methods in natural language processing-Volume 10, pp. 79-86, Association for Computational Linguistics, 2002.

[25] J. M. Wiebe, R. F. Bruce, and T. P. O'Hara, "Development and use of a gold-standard data set for subjectivity classifications," in Proceedings of the 37th annual meeting of the Association for Computational Linguistics on Computational Linguistics, pp. 246-253, Association for Computational Linguistics, 1999.

[26] P. Přibáň, T. Hercig, and L. Lenc, "Uwb at semeval-2018 task 1: Emotion intensity detection in tweets," in Proceedings of The 12th International Workshop on Semantic Evaluation, pp. 133-140, 2018. 
[27] R. K. Gupta and Y. Yang, "Crystalfeel at semeval-2018 task 1: Understanding and detecting emotion intensity using affective lexicons," in Proceedings of The 12th International Workshop on Semantic Evaluation, pp. 256-263, 2018.

[28] Z.-Q. Wang, X. Sun, D.-X. Zhang, and X. Li, "An optimal svm-based text classification algorithm," in 2006 International Conference on Machine Learning and Cybernetics, pp. 1378-1381, IEEE, 2006.

[29] R.-E. Fan, K.-W. Chang, C.-J. Hsieh, X.-R. Wang, and C.-J. Lin, "Liblinear - a library for large linear classification," 2008. The Weka classifier works with version 1.33 of LIBLINEAR.

[30] M. Hall, E. Frank, G. Holmes, B. Pfahringer, P. Reutemann, and I. H. Witten, "The weka data mining software: an update," ACM SIGKDD explorations newsletter, vol. 11, no. 1, pp. 10-18, 2009.

[31] A. Bagavathi, V. Rao, and A. A. Tzacheva, "Data distribution method for scalable actionable pattern mining," in Proceedings of the First International Conference on Data Science, E-learning and Information Systems, p. 3, ACM, 2018.

[32] Z. W. Ras, A. Dardzinska, L.-S. Tsay, and H. Wasyluk, "Association action rules," in Data Mining Workshops, 2008. ICDMW'08. IEEE International Conference on, pp. 283-290, IEEE, 2008.

[33] A. Tzacheva, J. Ranganathan, and S. Y. Mylavarapu, "Actionable pattern discovery for tweet emotions (in press)," in Proceedings of 10th International Conference on Applied Human Factors and Ergonomics, pp. 1-9, 2019. 